\title{
Predicting Risk of Potentially Preventable Hospitalization in Older Adults with Dementia
}

\author{
Donovan T. Maust, MD, MS, *广 (10 H. Myra Kim, ScD, ${ }^{*+}$ Claire Chiang, PhD, ** \\ Kenneth M. Langa, MD, PhD, ${ }^{\dagger \$}$ al and Helen C. Kales, MD*广
}

OBJECTIVES: Reducing potentially preventable hospitalization (PPH) among older adults with dementia is a goal of Healthy People 2020, yet no tools specifically identify patients with dementia at highest risk. The objective was to develop a risk prediction model to identify older adults with dementia at high imminent risk of PPH.

DESIGN: A 30-day risk prediction model was developed using multivariable logistic regression. Patients from fiscal years (FY) 2009 to 2011 were split into development and validation cohorts; FY2012 was used for prediction.

SETTING: Community-dwelling older adults ( $\geq 65$ years of age) with dementia who received care through the Veterans Health Administration.

PARTICIPANTS: There were 1793783 participants.

MEASUREMENTS: Characteristics associated with hospitalization risk were (1) age and other demographic factors; (2) outpatient, emergency department, and inpatient utilization; (3) medical and psychiatric diagnoses; and (4) prescribed medication use including changes to psychotropic medications (eg, initiation or dosage increase). Model discrimination was determined by the $\mathrm{C}$ statistic for each of the three cohorts. Finally, to determine whether predicted 30-day risk strata were stable over time, the observed PPH rate was calculated out to 1 year.

RESULTS: In the development cohort, $.6 \%$ of patients experienced PPH within 30 days. The $\mathrm{C}$ statistic for the development cohort was $.83(95 \%$ confidence interval $[\mathrm{CI}]=.83-.84)$ and .83 in the prediction cohort $(95 \% \mathrm{CI}=.82-.84)$. Patients in

From the "Department of Psychiatry, University of Michigan, Ann Arbor, Michigan; ${ }^{\dagger}$ Institute for Healthcare Policy and Innovation, University of Michigan, Ann Arbor, Michigan; ${ }^{*}$ Center for Clinical Management Research, VA Ann Arbor Healthcare System, Ann Arbor, Michigan; ${ }^{\S}$ Department of Internal Medicine, University of Michigan, Ann Arbor, Michigan; and the "Institute for Social Research, University of Michigan, Ann Arbor, Michigan.

Address correspondence to Donovan T. Maust, MD, MS, Department of Psychiatry, University of Michigan, 2800 Plymouth Road, NCRC

016-226W, Ann Arbor, MI 48109. E-mail: maustd@umich.edu; Twitter: @dtmaust

DOI: $10.1111 /$ jgs.16030 the top $10 \%$ of predicted 30 -day PPH risk accounted for more than $50 \%$ of 30-day PPH admissions in all three cohorts. In addition, those predicted to be at elevated 30-day risk remained at higher risk throughout a year of follow-up.

CONCLUSION: It is possible to identify older adults with dementia at high risk of imminent $\mathrm{PPH}$, and their risk remains elevated for an entire year. Given the negative outcomes associated with acute hospitalization for those with dementia, healthcare systems and providers may be able to engage these high-risk patients proactively to avoid unnecessary hospitalization. J Am Geriatr Soc 67:2077-2084, 2019.

Key words: dementia; potentially preventable hospitalization; risk prediction

$\mathrm{P}$ atients with dementia have an all-cause hospitalization rate approximately 1.4 times higher than other older adults, and potentially preventable hospitalization $(\mathrm{PPH})$ is nearly 1.8 -times higher. ${ }^{1} \mathrm{PPH}$ captures admission for ambulatory care-sensitive conditions such as congestive heart failure (CHF) or pneumonia, that, with optimal outpatient access and management, are potentially unnecessary. Reduction of PPH specifically in older adults with dementia is a goal of Healthy People 2020., ${ }^{2,3}$ Their elevated hospitalization risk is worrying because, although all older adults are at increased risk of hospitalization-associated delirium, iatrogenic complications, and cognitive and functional decline, ${ }^{4-6}$ the consequences are greater for patients with dementia, ${ }^{7,8}$ for whom cognitive or functional decline are risk factors for institutionalization., ${ }^{9,10}$

As the population with dementia nearly triples by $2050,{ }^{11}$ even small reductions in the rate of PPH could have a large impact. Unfortunately, no controlled dementia care intervention trials have demonstrated a reduction in hospitalization. ${ }^{12,13}$ One possible reason is the trials were not specifically designed to target patients at the highest risk of hospitalization. Given the potential adverse consequences of hospitalization for older adults with dementia, identifying 
those at high risk before they need to be hospitalized, especially admissions for conditions that could potentially be treated in an outpatient setting, is critical.

Approaches to risk-stratify patients with dementia that do not rely on overburdened primary care clinicians ${ }^{14}$ is key to appropriately targeting supports that may benefit these older adults and their caregivers. ${ }^{15}$ For this analysis, we used national data from the US Veterans Health Administration (VHA) electronic health record (EHR) to develop a multivariable logistic regression model to predict PPH admission within 30 days (developmental cohort). We then used the model to identify risk tiers among a different cohort of older adults with dementia (validation cohort) and determine whether the model could accurately predict risk among the new set of patients (prediction cohort).

\section{METHODS}

\section{Study Sample and Outcome}

The study sample ( $\mathrm{n}=1793$ 783) was drawn from older adults treated in the VHA from October 1, 2008, through September 30, 2012 (fiscal years [FY] 2009-2012). The first index date was October 1, 2008 (ie, the start of FY2009), with a cohort including patients who met these four inclusion criteria: (1) 65 years of age or older; (2) at least one inpatient or outpatient encounter within the previous 12 months to establish use of VHA services; (3) dementia diagnosis before index date (based on one or more encounter with one of the following International Classification of Diseases, Ninth Revision, Clinical Modification (ICD-9-CM) codes, as used in previous work ${ }^{16-18}$ : 046.1, 046.3, 290.0, 290.1x, 290.2x, 290.3, 290.4x, 291.2, 294.10, 294.11, 331.0, 331.1, and 331.82); and (4) not in a VHA inpatient or long-term care setting on the index date.

Then we expanded the cohort by moving the index date ahead at 2-month intervals from December 1, 2008; February 1, 2009; and so on, through August 1, 2012. All patients who met the four inclusion criteria at the index date of each 2-month interval (eg, on December 1, 2008) were considered at risk for PPH and included in the cohort. Therefore, a single patient could contribute multiple at-risk intervals to the final cohort.

The event of interest was PPH admission within 30 days of entering the cohort (ie, the index date). We defined PPH using the primary ICD-9-CM discharge diagnosis (Table S1) for the inpatient admission, applying the Agency for Healthcare Research and Quality (AHRQ) prevention quality indicators ${ }^{19}$ that include conditions both acute (eg, dehydration, bacterial pneumonia, kidney or urinary tract infection) and chronic (diabetes, asthma, chronic obstructive pulmonary disease, hypertension, CHF exacerbation, angina). To be consistent with prior studies, ${ }^{1,3,20}$ we also included cellulitis, gastric/duodenal/peptic ulcer, ear/ nose/throat infection, gastroenteritis, hypoglycemia, hypokalemia, influenza, malnutrition, and seizure disorder. The total cohort from the first 3 years (FY2009-2011) was randomly split into halves to develop and validate the prediction model ( $\mathrm{n}=664355$ and 664357 , respectively); the final year (FY2012; $\mathrm{n}=465$ 071) was used as a prediction cohort to apply the model.

\section{Measures}

Candidate model variables were chosen based on prior work examining predictors of hospitalization in older adults. ${ }^{21-23}$ Demographic variables included age, sex, race (white, black, other race, unknown race), Hispanic ethnicity, marital status (married, single/never married, divorced, widowed, unknown), and urbanicity (urban, rural, highly rural as defined by VHA using US Census designations). Clinical characteristics included length of time since the first dementia diagnosis (a proxy for dementia severity, ascertained from records going back $10 \mathrm{y}$ ), number of unique prescription medications as of the index date, and presence of the following conditions based on clinical encounters in the preceding 12 months (Table S1): diagnoses used to derive the Charlson Comorbidity Index; each PPH condition; delirium (or other transient mental status change); and individual psychiatric conditions including depression, bipolar disorder, and anxiety disorders.

Service utilization characteristics included the number of outpatient, inpatient, and emergency department visits for specific pre-index intervals: $1,2,3,6,9$, and 12 months.

Behavioral and psychological symptoms of dementia may be associated with hospitalization risk, ${ }^{24,25}$ and the use of psychotropic medications suggests these symptoms are present, ${ }^{26}$ so we used indicators of psychotropic (eg, antipsychotic, antidepressant, sedative/hypnotic, mood stabilizer) and antidementia (eg, cholinesterase inhibitors and memantine) medication prescribing: prevalent use, incident use, and dosage escalation. Each psychotropic indicator was determined for 1, 2, 3, 6, 9 , and 12 months pre-index, as well as on the index date.

\section{Analysis}

Our goal was to develop a model predicting 30-day PPH risk; we followed an analytic plan similar to one used to develop and validate a suicide risk prediction model among VHA patients using the VHA EHR. ${ }^{27}$ To develop a model to predict 30-day PPH, we used the development cohort and fit multivariable logistic regression. Generalized estimation equation with independence was used to adjust for potential correlation from repeated inclusion of the same patients.

Because the medication and service utilization measures were collected for several prespecified time intervals that were potentially correlated (eg, antipsychotic use 1, 2, 3, 6, 9 , and 12 months pre-index), we screened each set of measures separately to determine which were most predictive. Based on the magnitude and significance of the parameter estimates, 1 month pre-index was most predictive of $\mathrm{PPH}$, so the final model only included medication and service utilization indicators from the month pre-index. Longer preindex intervals (eg, 3 or 6 months pre-index) did not further add to model predictiveness, with parameter estimates close to zero.

To allow for effect modification of diagnoses and utilization measures by patient age, we also considered variable-by-age interaction terms, but they were neither significant nor improved the model fit so were not retained. For continuous or count measures (age, number of outpatient visits, etc), we included linear and square terms after centering the measures to allow nonlinear relationships. We did not use any additional variable reduction approaches and retained all variables in the model regardless of statistical significance 
because our focus was on overall prediction of risk rather than causal inferences related to specific characteristics and the associated PPH risk.

We present the distribution of select patient sociodemographic and clinical characteristics in the development cohort overall and among those with a PPH admission within 30 days or 1 year. Patient characteristics are described for those at risk of PPH at all intervals; if a patient is at risk of $\mathrm{PPH}$ in more than one interval, that person's contribution to the patient characteristics is considered as if from a different patent for each interval. Therefore, the values of the predictors corresponding to each time interval are all accounted for separately.

Model discrimination was assessed by calculating the $\mathrm{C}$ statistic in each of the three cohorts: development (half 1 of FY2009-2011), validation (half 2 of FY2009-2011), and prediction (FY2012). In each study cohort, based on the ranked predicted probability of $\mathrm{PPH}$ admission within 30 days, we set risk cut points starting with those patients with the top $.1 \%$ predicted probability down through lower, more inclusive tiers of risk (eg, top $.5 \%, 1.0 \%, 5.0 \%$, etc). We examined risk concentration for each cut point by determining the number of observed PPH admissions for patients in the risk tier divided by the number of expected PPH admissions based on the overall cohort PPH rate. We used the validation cohort to display calibration graphically by deciles of predicted risk and also used the Hosmer-Lemeshow test for goodness of fit.

To appreciate the impact of applying the model to a new set of patients, performance characteristics (sensitivity, specificity, positive predictive value, and negative predictive value) were determined in the prediction cohort using the prespecified risk cut points.

Finally, to determine whether those at high imminent (ie, 30-day) risk of PPH remained at high risk throughout an entire year, we applied the 30-day PPH risk cut points to the prediction cohort to determine each tier's risk concentration 1, 3, 6, and 12 months post-index. Statistical analyses were done using SAS v.9.4 (SAS Institute, Cary, NC).

\section{RESULTS}

Select demographic and clinical characteristics of the development cohort are presented in Table 1 (all characteristics are listed in Table S2) that included 664355 adults with dementia. The 30-day PPH rate was 69.7 per 1000 personyears; 1-year PPH rate was 57.6 per 1000 person-years. The PPH rate of black patients was nearly double that of white patients. Those patients who experienced PPH within 30 days had more use of each type of inpatient and outpatient service, as well as more of every category of psychotropic medication use including dose increases in the preindex month.

The development model had very good discrimination, ${ }^{28}$ with a C statistic $=.834(95 \%$ confidence interval $[\mathrm{CI}]=.827-.840)$; model coefficients are presented in Table S3. When the model was applied to the validation and prediction cohorts, there was only a slight loss of discrimination, with $\mathrm{C}$ statistics of $.832(95 \% \mathrm{CI}=.825-.838)$ and $.829(95 \%$ $\mathrm{CI}=.821-.838)$, respectively. Figure 1 plots the expected (predicted) and observed PPH admission rates by decile of predicted risk for the validation cohort. The predicted rate was close to the observed rate across deciles, although the model slightly overestimated events in the lower deciles and slightly underestimated among the highest risk groups. Although the Hosmer-Lemeshow statistic was significant $\left(\chi^{2}=159.8 ; \mathrm{df}=8\right.$; $P<.001)$, this is likely a function of the large sample size.

In each of the three cohorts of patients with dementia (Table 2), among the top $1.0 \%$ of patients by predicted 30-day PPH probability, the observed PPH rate ranged from 13.4 to 14.1 times higher than the crude rates (ie, risk concentration). Little overfitting was indicated as seen by the consistent risk concentration across all three cohorts for each predicted probability cut point. Of note, patients in the top $10 \%$ of predicted 30 -day PPH risk accounted for $52.9 \%$ to $53.9 \%$ of 30 -day PPH admissions in each cohort. Performance characteristics for various cut points of predicted risk are presented in Table 3.

Finally, we applied our predicted 30-day probability cut points to the prediction cohort to examine the trajectory of risk (ie, observed PPH number, rate, and risk concentration) over 1 year (Figure 2 and Table S4). Older adults predicted to be at high 30-day risk of PPH had an elevated risk of admission for the entire year. For example, the top $1 \%$ had 13.4-times higher 30-day PPH risk than patients with dementia overall during the first month, but over the following year their 12-month PPH risk was still 7.7-times higher than the 12-month risk for patients with dementia overall.

\section{DISCUSSION}

Using data from more than 660000 adults with dementia, we developed a model that showed high discrimination for predicting 30-day risk of PPH using information available in the EHR. The model also had very good discrimination in the validation cohort $(\mathrm{C}$ statistic $=.83)$, indicating little overfitting. Discrimination of the prediction model in the prediction cohort was also high $(\mathrm{C}$ statistic $=.83)$, and prediction cohort patients at high 30-day risk remained at elevated risk during 1 year of follow-up. Although this model needs to be tested in other health systems to determine generalizability, our findings suggest this EHR-based approach may be feasible.

We found a PPH risk gradient among older adults with dementia: within the top $.1 \%, 30$-day risk of PPH was nearly 20 times higher than among patients overall. Expanding the definition of high risk to the top $10 \%$, a fivefold higher risk of 30-day PPH remained. Although risk concentration did decrease over 12 months, those at high 30-day risk maintained persistently elevated risk. A variety of dementia care management and caregiver support programs decreased caregiver burden, patient behavioral symptoms of dementia, or time to nursing home placement, ${ }^{29-32}$ but intervention trials typically did not reduce hospitalization. ${ }^{33}$ Null findings from randomized intervention trials may suggest these interventions do not reduce hospitalization; an alternative explanation is that participants were not riskstratified to maximize hospitalization impact. The GRACE intervention trial, which provided care management for low-income older adults (with or without dementia), demonstrated this: there was no impact on hospitalization overall, but admissions were reduced in those who screened at high risk of hospitalization at baseline. ${ }^{34}$ Given the growing 
Table 1. Select Demographic and Clinical Characteristics and 30-day and 1-year Rates of Potentially Preventable Hospitalization among the Development Cohort

\begin{tabular}{|c|c|c|c|c|c|c|c|c|}
\hline \multirow{2}{*}{$\begin{array}{l}\text { Characteristic }^{\mathrm{a}} \\
\text { All }\end{array}$} & \multicolumn{2}{|c|}{$\mathrm{N}(\%)$} & \multicolumn{2}{|c|}{$\begin{array}{l}\text { Patients with PPH } \\
\text { within } 30 \mathrm{~d}, \mathrm{~N}(\%)\end{array}$} & \multicolumn{2}{|c|}{$\begin{array}{l}\text { Patients with PPH } \\
\text { within } 1 \text { y, N (\%) }\end{array}$} & \multirow{2}{*}{$\begin{array}{c}\begin{array}{c}\text { 30-d PPH rate } \\
\text { per 1000 } \\
\text { person-years }\end{array} \\
69.7\end{array}$} & \multirow{2}{*}{$\begin{array}{c}\begin{array}{c}1-y \text { PPH rate } \\
\text { per } 1000 \\
\text { person-years }\end{array} \\
57.6\end{array}$} \\
\hline & 664355 & & 3745 & (.6) & 31962 & $(4.8)$ & & \\
\hline \multicolumn{9}{|l|}{ Age, y } \\
\hline $65-74$ & 113964 & $(17.2)$ & 659 & $(17.6)$ & 5732 & $(17.9)$ & 71.5 & 58.9 \\
\hline $75-84$ & 322642 & (48.6) & 1688 & $(45.1)$ & 14639 & $(45.8)$ & 64.6 & 53.6 \\
\hline$\geq 85$ & 227749 & (34.3) & 1398 & $(37.3)$ & 11591 & $(36.3)$ & 76.1 & 63.0 \\
\hline \multicolumn{9}{|l|}{ Sex } \\
\hline Male & 647274 & (97.4) & 3646 & $(97.4)$ & 31088 & $(97.3)$ & 69.7 & 57.6 \\
\hline \multicolumn{9}{|l|}{ Race } \\
\hline White & 482594 & (72.6) & 2743 & $(73.2)$ & 23214 & $(72.6)$ & 70.3 & 57.5 \\
\hline Black & 67907 & (10.2) & 694 & (18.5) & 5971 & (18.7) & 127.1 & 109.6 \\
\hline Other races & 15463 & (2.3) & 100 & (2.7) & 943 & (3.0) & 80.0 & 73.4 \\
\hline Unknown or missing & 98391 & (14.8) & 208 & $(5.6)$ & 1834 & $(5.7)$ & 26.1 & 22.0 \\
\hline \multicolumn{9}{|l|}{ Hispanic ethnicity } \\
\hline Yes & 17794 & (2.7) & 156 & $(4.2)$ & 1236 & (3.9) & 108.8 & 85.1 \\
\hline No & 575614 & (86.6) & 3421 & (91.4) & 29277 & (91.6) & 73.5 & 60.9 \\
\hline Unknown or missing & 70947 & (10.7) & 168 & (4.5) & 1449 & $(4.5)$ & 29.3 & 24.5 \\
\hline \multicolumn{9}{|l|}{ Marital status } \\
\hline Married & 429644 & (64.7) & 1903 & $(50.8)$ & 16399 & $(51.3)$ & 54.7 & 45.0 \\
\hline Single/never married & 35670 & $(5.4)$ & 294 & $(7.9)$ & 2586 & $(8.1)$ & 102.6 & 90.0 \\
\hline Divorced & 79610 & (12.0) & 651 & (17.4) & 5440 & $(17.0)$ & 101.5 & 84.0 \\
\hline Widowed & 116469 & (17.5) & 889 & $(23.7)$ & 7465 & $(23.4)$ & 94.7 & 79.4 \\
\hline Unknown or missing & 2962 & (.4) & 8 & (.2) & 72 & (.2) & 33.4 & 29.2 \\
\hline \multicolumn{9}{|l|}{ Residence } \\
\hline Urban & 432406 & $(65.1)$ & 2670 & $(71.3)$ & 22558 & $(70.6)$ & 76.4 & 62.9 \\
\hline Rural & 224684 & (33.8) & 1048 & $(28.0)$ & 9159 & $(28.7)$ & 57.6 & 48.2 \\
\hline Highly rural & 7265 & (1.1) & 27 & (.7) & 245 & $(.8)$ & 45.9 & 39.8 \\
\hline \multicolumn{9}{|l|}{ Diagnoses present in past $12 \mathrm{mo}$} \\
\hline Myocardial infarction & 20181 & $(3.0)$ & 337 & $(9.0)$ & 2182 & $(6.8)$ & 210.0 & 144.1 \\
\hline Cerebrovascular disease & 125495 & (18.9) & 1256 & (33.5) & 10227 & $(32.0)$ & 124.7 & 102.9 \\
\hline $\begin{array}{l}\text { Delirium or other transient } \\
\text { mental status change }\end{array}$ & 280286 & (42.2) & 2100 & $(56.1)$ & 16953 & $(53.0)$ & 93.0 & 74.5 \\
\hline Depression & 167864 & (25.3) & 1249 & (33.4) & 10508 & (32.9) & 92.2 & 76.3 \\
\hline Posttraumatic stress disorder & 40426 & $(6.1)$ & 309 & (8.3) & 2545 & $(8.0)$ & 94.7 & 76.3 \\
\hline Other anxiety disorders & 48414 & (7.3) & 413 & $(11.0)$ & 3295 & (10.3) & 105.9 & 83.2 \\
\hline \multicolumn{9}{|l|}{ Duration of dementia, $y$} \\
\hline$<1$ & 165081 & (24.8) & 1035 & $(27.6)$ & 7811 & $(24.4)$ & 77.7 & 56.8 \\
\hline $1-3$ & 292349 & (44.0) & 1510 & $(40.3)$ & 13327 & $(41.7)$ & 63.8 & 54.4 \\
\hline $4-6$ & 141122 & (21.2) & 709 & (18.9) & 6645 & $(20.8)$ & 62.1 & 56.3 \\
\hline$\geq 7$ & 65803 & $(9.9)$ & 491 & (13.1) & 4179 & (13.1) & 92.4 & 77.1 \\
\hline \multicolumn{9}{|l|}{ Type of dementia } \\
\hline Alzheimer's disease & 545775 & (82.2) & 2794 & $(74.6)$ & 24050 & $(75.2)$ & 63.3 & 52.6 \\
\hline Vascular dementia & 175687 & (26.4) & 1429 & $(38.2)$ & 12212 & $(38.2)$ & 101.0 & 85.8 \\
\hline Lewy body dementia & 32098 & $(4.8)$ & 236 & (6.3) & 1762 & $(5.5)$ & 91.6 & 69.9 \\
\hline $\begin{array}{l}\text { Progressive multifocal } \\
\text { leukoencephalopathy }\end{array}$ & 256 & $(<.1)$ & 2 & $(.1)$ & 11 & $(<.1)$ & 97.2 & 52.3 \\
\hline Jakob-Creutzfeldt disease & 236 & $(<.1)$ & 3 & $(.1)$ & 21 & $(.1)$ & 157.9 & 109.4 \\
\hline Alcoholic dementia & 20558 & (3.1) & 167 & (4.5) & 1419 & (4.4) & 101.0 & 85.4 \\
\hline Pick's dementia & 9980 & (1.5) & 59 & (1.6) & 533 & (1.7) & 73.1 & 64.4 \\
\hline \multicolumn{9}{|l|}{ Utilization in past month } \\
\hline \multicolumn{9}{|c|}{ Medical/surgical outpatient visits, $n$} \\
\hline 0 & 336372 & (50.6) & 732 & (19.6) & 7846 & $(24.5)$ & 26.8 & 27.1 \\
\hline 1 & 147262 & (22.2) & 687 & (18.3) & 6753 & $(21.1)$ & 57.5 & 54.1 \\
\hline 2 & 77930 & (11.7) & 564 & (15.1) & 5037 & $(15.8)$ & 89.6 & 78.4 \\
\hline$\geq 3$ & 102791 & (15.5) & 1762 & (47.1) & 12326 & (38.6) & 216.1 & 162.1 \\
\hline
\end{tabular}


Table 1 (Contd.)

\begin{tabular}{|c|c|c|c|c|c|c|c|c|}
\hline$\frac{\text { Characteristic }^{\mathrm{a}}}{\text { Acute inpatient hospitalizations, } \mathrm{n}}$ & & & & & & & $\begin{array}{l}\text { 30-d PPH rate } \\
\text { per } 1000 \\
\text { person-years }^{b}\end{array}$ & $\begin{array}{l}\text { 1-y PPH rate } \\
\text { per } 1000 \\
\text { person-years }^{b}\end{array}$ \\
\hline 0 & 655674 & $(98.7)$ & 3314 & (88.5) & 29992 & (93.8) & 62.4 & 54.5 \\
\hline 1 & 8313 & (1.3) & 396 & (10.6) & 1851 & (5.8) & 640.5 & 403.9 \\
\hline$\geq 2$ & 368 & $(.1)$ & 35 & (.9) & 119 & $(.4)$ & 1412.7 & 824.0 \\
\hline 0 & 139929 & $(21.1)$ & 517 & (13.8) & 4178 & (13.1) & 45.9 & 36.8 \\
\hline $1-2$ & 93255 & (14.0) & 265 & $(7.1)$ & 2675 & $(8.4)$ & 35.0 & 33.4 \\
\hline $3-4$ & 114498 & (17.2) & 348 & (9.3) & 3678 & (11.5) & 37.4 & 37.1 \\
\hline $5-6$ & 109603 & (16.5) & 537 & (14.3) & 4548 & (14.2) & 60.4 & 48.4 \\
\hline$\geq 7$ & 207070 & (31.2) & 2078 & $(55.5)$ & 16883 & (52.8) & 124.6 & 100.6 \\
\hline \multicolumn{9}{|c|}{ Class of psychotropic use on index date } \\
\hline \multicolumn{9}{|c|}{ Psychotropic dose increase in prior month } \\
\hline Antipsychotic & 19709 & (3.0) & 178 & $(4.8)$ & 1430 & $(4.5)$ & 112.4 & 92.6 \\
\hline Antidepressant & 51758 & $(7.8)$ & 411 & $(11.0)$ & 3439 & $(10.8)$ & 98.4 & 80.7 \\
\hline Sedative/Hypnotic & 13970 & $(2.1)$ & 121 & $(3.2)$ & 952 & $(3.0)$ & 107.4 & 83.6 \\
\hline Mood stabilizer & 13526 & $(2.0)$ & 137 & $(3.7)$ & 1108 & (3.5) & 125.8 & 101.6 \\
\hline
\end{tabular}

Abbreviation: PPH, potentially preventable hospitalization.

${ }^{\text {a } F o r ~ t h e ~ c o m p l e t e ~ l i s t ~ o f ~ c h a r a c t e r i s t i c s ~ i n c l u d e d ~ i n ~ t h e ~ p r e d i c t i v e ~ m o d e l ~ a n d ~ m o d e l ~ c o e f f i c i e n t s, ~ s e e ~ T a b l e ~ S 2 ~ a n d ~ T a b l e ~ S 3, ~ r e s p e c t i v e l y . ~}$

${ }^{\mathrm{b}}$ Calculated as number of patients with PPH divided by the at-risk days where person-days are counted until the earliest date of PPH, non-PPH admission, death, or end of follow-up period ( $30 \mathrm{~d}$ for $30-\mathrm{d}$ rate and $12 \mathrm{mo}$ for 12 -mo rate) and expressed as per 1000 person-years. Participants who were at risk in multiple intervals are counted multiple times. In the development cohort, $16396(13 \%)$ patients were included only once, $15042(12 \%)$ patients twice, 14 $049(11.2 \%)$ patients 3 times, $12868(10.2 \%)$ patients 4 times, $11567(9.2 \%)$ patients 5 times, $10921(8.7 \%)$ patients 6 times, $10714(8,5 \%) 7$ times, 10 $066(8 \%) 8$ times, $8921(7.1 \%)$ patients 9 times, $6693(5.3 \%) 10$ times, $4434(3.5 \%) 11$ times, $2386(1.9 \%)$ patients 12 times, $1092(.8 \%)$ patients 13 times, $358(.3 \%)$ patients 14 times, $84(.07 \%)$ patients 15 times, $14(.01 \%)$ patients 16 times, 2 patients 17 times, and 1 patient 18 times.

population of older adults with dementia, limited resources call for risk stratification strategies to help target interventions appropriately, rather than attempting to deliver a given intervention to all.

Analyses based on claims or administrative data of older adults can be subject to unobserved confounding by factors such as frailty or functional status that are associated with health outcomes but not routinely available in administrative data. ${ }^{35,36}$ Yet our model had excellent predictive ability derived entirely from structured information in the EHR, without any additional information from the patient or caregiver. In contrast, the Probability of Repeated Admission Instrument, used in the GRACE trial to identify the highrisk patients at baseline, has to be completed by the older adult and includes items on self-rated health and the presence of an informal caregiver. ${ }^{37}$ Given the enormous demands on primary care providers' time ${ }^{14}$ harnessing the EHR may be a feasible means to risk-stratify these patients without requiring any additional input from providers, patients, or caregivers.

The ability of our model to identify high-risk patients is notable given that the overall cohort, older adults with dementia, is, at baseline, at a significantly elevated risk of admission compared with the general adult population. The predictive ability of our model is comparable with a separately developed PPH predictive model for VA patients of all ages, ${ }^{23}$ but our model discriminates slightly better than other risk models specifically developed for older adults. ${ }^{22,38-41}$ A review of risk stratification applied six different models: Adjusted Clinical
Groups, Hierarchical Condition Categories, Elder Risk Assessment, Chronic Comorbidity Count, Charlson Comorbidity Index, and Minnesota Health Care Home Tiering to more than 80000 primary care patients seen in a large academic health system. Across the six models, the $\mathrm{C}$ statistic for 1-year hospitalization prediction was .67 to $.73 .^{39}$

One specific model feature that may have facilitated identifying high-risk patients was the inclusion of psychotropic medications as predictors, specifically new medication starts and dosage increases. Changes to psychotropic medications may herald the presence or worsening of symptoms such as agitation or psychosis. ${ }^{26,42}$ Such behavioral and psychological symptoms of dementia may dominate the clinical presentation of patients with dementia, and the related caregiver distress is associated with increased hospitalization and costs for patients. ${ }^{25}$ Alternatively, such medication changes may be to treat delirium or other transient changes in mental status that were recorded for more than $40 \%$ of cohort patients in the prior 12 months. Although information about behavioral symptoms is typically not available in the EHR or delirium may not be reliably recorded, incorporating information about psychotropic medication changes, which would be in the EHR, may help identify patients whose behavioral problems either reflect a worsening medical problem or are part of the constellation of symptoms that led to hospital admission. ${ }^{42}$ Regardless of the clinical rationale for such medication changes, this information can be useful to identify high-risk older adults. 


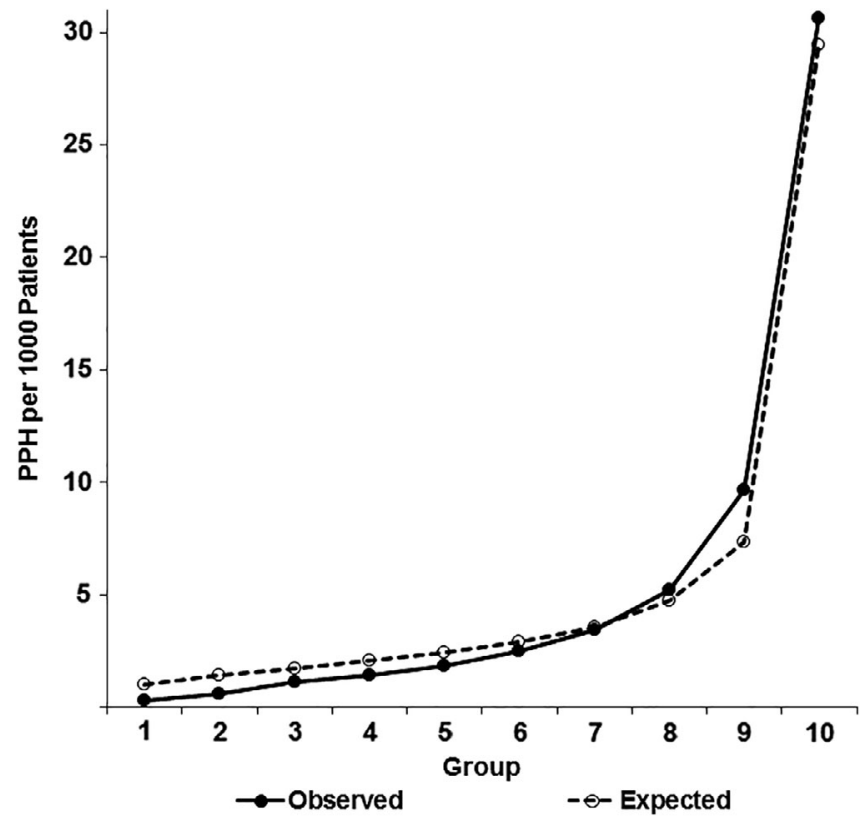

Figure 1. Calibration curve for the predicted 30-day potentially preventable hospitalization (PPH) risk model applied to the validation cohort. The figure compares observed and expected (predicted) PPH admissions across deciles of risk among older adults with dementia in the Veterans Health Administration. Expected events closely follow observed events, although given the large sample size, prediction does vary across deciles, slightly overpredicting risk in the bottom deciles and slightly underpredicting risk in the top deciles (Hosmer-Lemeshow statistic: $\left.\chi^{2}=159.8 ; \mathrm{df}=8 ; P<.001\right)$.

The 12-month PPH admission rate among our population57.6 admissions per 1000 person-years in the development sample-is, as expected for patients with dementia, higher than for adults overall in the $\mathrm{VHA}^{43}$ and older adults in the
Table 3. Performance Characteristics by Cut Point of Predicted 30-day PPH Risk in the Prediction Cohort

\section{Predicted probability}

cut point ${ }^{\mathrm{a}}, \%$

Sensitivity, \% $\quad$ Specificity, \% $\quad$ PPV, $\% \quad$ NPV, $\%$

\begin{tabular}{lrrrr}
\hline Top .1 & 1.9 & 99.9 & 9.9 & 99.5 \\
Top .5 & 7.7 & 99.5 & 8.1 & 99.5 \\
Top 1.0 & 13.4 & 99.1 & 7.1 & 99.5 \\
Top 5.0 & 37.4 & 95.2 & 3.9 & 99.7 \\
Top 10.0 & 52.9 & 90.2 & 2.8 & 99.7 \\
Top 20.0 & 69.3 & 80.3 & 1.8 & 99.8 \\
Top 50.0 & 90.3 & 50.2 & 1.0 & 99.9 \\
100.0 & 100.0 & .0 & .5 & NA \\
\hline
\end{tabular}

Abbreviations: NA, due to 0 denominator; NPV, negative predictive value; $\mathrm{PPH}$, potentially preventable hospitalization; PPV, positive predictive value.

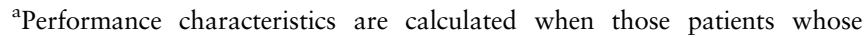
predicted 30-day PPH risk probability is at or exceeds the row cut point are considered positive for $\mathrm{PPH}$ and the remaining patients are considered negative for PPH.

general population. ${ }^{44}$ However, the rate is lower than in an analysis of Medicare beneficiaries with dementia. ${ }^{20}$ This discrepancy between VHA and Medicare PPH rates is partially because most older VHA patients have Medicare, so some PPH admissions occur at community facilities. Although this analysis was not designed to identify particular characteristics associated with PPH, it is notable that black patients experienced much higher PPH rates than other patients, in a system designed for equal access to care regardless of socioeconomic or insurance status.

A limitation of our analysis is that it only includes data from the VHA. Although this limits generalizability, it suggests feasibility for other healthcare systems to develop and implement their own internal risk prediction models. The model only captures PPH risk among patients identified with a dementia diagnosis that likely represents the subset

Table 2. The 30-day PPH Risk Concentration and Admission Rate by Cut Point of Predicted 30-day PPH Probability across the Three Cohorts

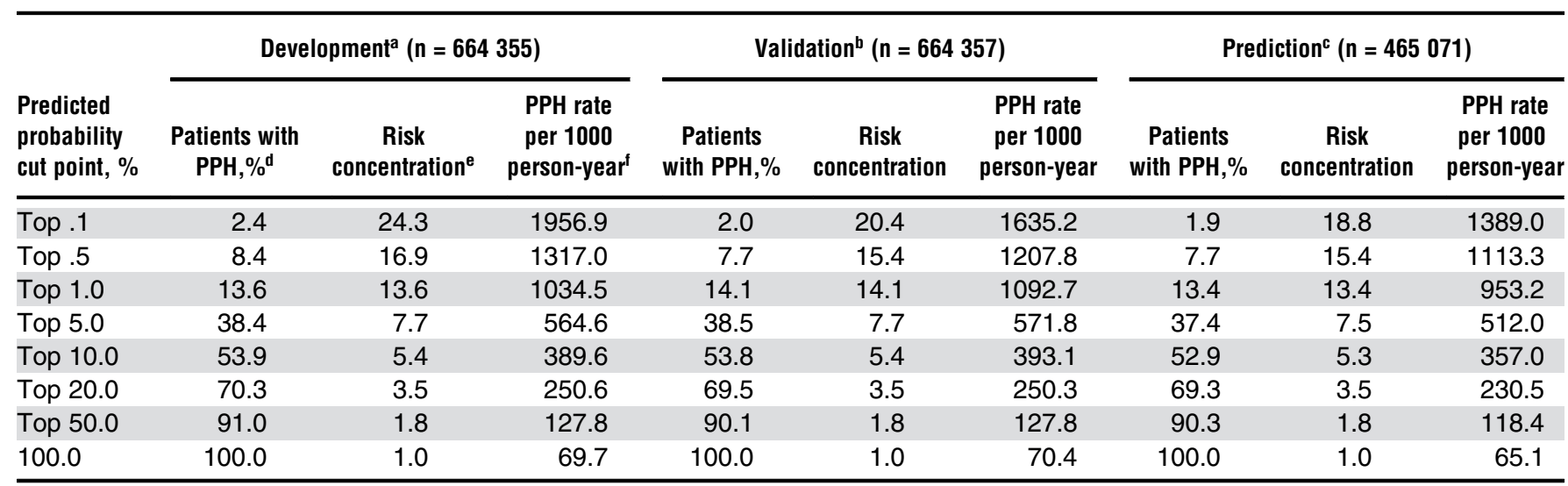

Abbreviation: $\mathrm{PPH}$, potentially preventable hospitalization.

${ }^{\mathrm{a} C}$ statistic $=.83 ; 95 \% \mathrm{CI}=.82-.84$.

${ }^{\mathrm{b}} \mathrm{C}$ statistic $=.83 ; 95 \% \mathrm{CI}=.82-.83$.

${ }^{\mathrm{c}} \mathrm{C}$ statistic $=.82 ; 95 \% \mathrm{CI}=.82-.83$.

${ }^{\mathrm{d}}$ The percentage of overall cohort PPH admissions accounted for by the patients within a given risk tier.

${ }^{\text {e}}$ Risk concentration = (observed no. of PPHs in risk tier)/(expected no. of PPHs based on rate among older adults with dementia overall).

${ }^{\mathrm{f}}$ Calculated as the number of patients with PPH for each cut point divided by the at-risk days where person-days are counted until the earliest date of PPH, non-PPH admission, death, or end of follow-up period ( $30 \mathrm{~d}$ ) and expressed as per 1000 person-years. 

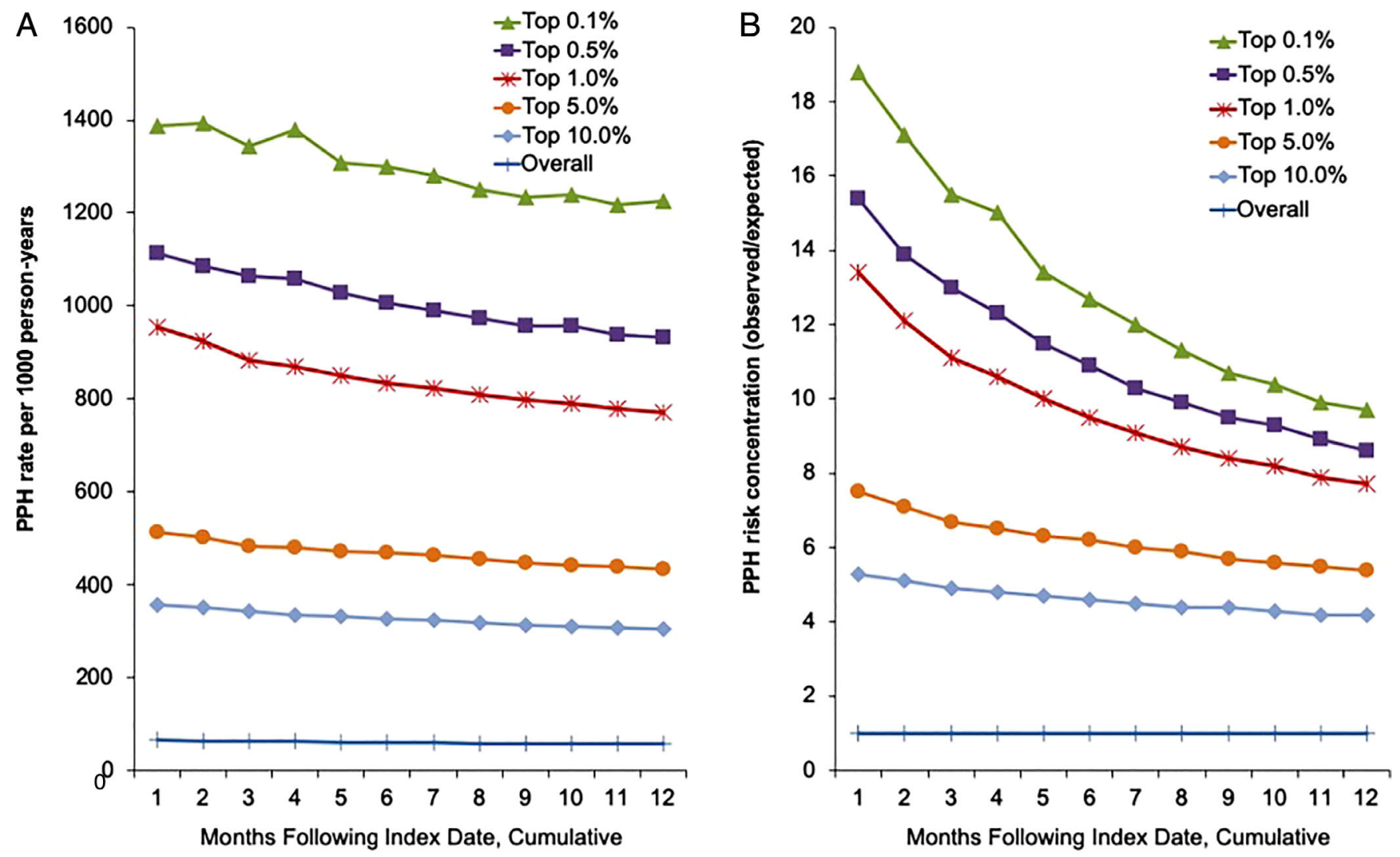

Figure 2. Cumulative potentially preventable hospitalization (PPH) rate and risk concentration over 1 year by cut point of 30-day predicted PPH risk in the prediction cohort. This figure demonstrates the change in A, PPH rate, and B, PPH risk concentration (observed PPH admissions in risk tier)/(predicted PPH admissions based on the rate among older adults with dementia overall) over 12 months based on the tier of predicted risk in the prediction cohort. Although the rate and risk concentration decline over time, the risk tiers maintain their relative positions at all time points, and those at elevated 30-day risk continue to experience PPH admissions at higher rates all year.

of patients with more advanced illness. In addition, the prediction data set is drawn from 2012 that is now more than 6 years old. In 2012, the VA introduced the Strategic Analytics for Improvement and Learning Value Model that includes PPH as a quality indicator. Heightened attention to PPH and other systemwide delivery changes mean a model developed with more recent data may perform differently. There is controversy over the utility of the PPH construct; a 2016 study found that, of AHRQ-designated preventable admissions, reviewing clinicians considered less than half preventable. ${ }^{45}$ However, Hodgson and colleagues suggest the PPH construct is still potentially useful, provided it is used to understand the underlying mechanisms increasing admission and not just to examine factors associated with admission. ${ }^{46}$ In this case, although risk prediction may help identify patients who could benefit from intervention, it cannot suggest the type of intervention that would be effective.

In conclusion, the acute inpatient hospital is a challenging environment for older adults with cognitive impairment, stressful to both patients and their caregivers. It is critical to address healthcare issues proactively before a crisis occurs and patients with dementia require hospitalization, particularly as the number of older adults with dementia grows. This analysis demonstrates that it is possible for healthcare systems to accurately identify older adults with dementia at high risk of imminent PPH.

\section{ACKNOWLEDGMENTS}

Financial Disclosure: Donovan T. Maust was supported by the Beeson Career Development Award Program (National Institute on Aging [NIA] K08AG048321, American Federation for Aging Research, the John A. Hartford Foundation, and the Atlantic Philanthropies) and R01AG056407. Kenneth M. Langa was supported by the NIA (P30AG053760, P30AG024824, R01AG053972).

Conflict of Interest: The authors have declared no conflicts of interest for this article.

Author Contributions: Study concept and design: Maust, Kim, Chiang, and Kales. Acquisition of subjects and/or data: Maust and Chiang. Analysis and interpretation of data: All authors. Preparation of manuscript: All authors.

Sponsor's Role: The funding source had no role in the design and conduct of the study; collection, management, analysis, and interpretation of the data; preparation, review, or approval of the manuscript; and decision to submit the manuscript for publication.

\section{REFERENCES}

1. Phelan EA, Borson S, Grothaus L, Balch S, Larson EB. Association of incident dementia with hospitalizations. JAMA. 2012;307(2):165-172. 
2. US Department of Health and Human Services, Office of Disease Prevention and Health Promotion. Healthy People 2020. 2020 Topics \& Objectives - Objectives A-Z. https://www.healthypeople.gov/2020/topics-objectives/topic/dementias-includingalzheimers-disease/objectives. Accessed June 11, 2019.

3. Culler SD, Parchman ML, Przybylski M. Factors related to potentially preventable hospitalizations among the elderly. Med Care. 1998;36(6):804-817.

4. Krumholz HM. Post-hospital syndrome-an acquired, transient condition of generalized risk. N Engl J Med. 2013;368(2):100-102.

5. Gillick MR, Serrell NA, Gillick LS. Adverse consequences of hospitalization in the elderly. Soc Sci Med. 1982;16(10):1033-1038.

6. Mathews SB, Arnold SE, Epperson CN. Hospitalization and cognitive decline: can the nature of the relationship be deciphered? Am J Geriatr Psychiatry. 2014;22(5):465-480.

7. Fick DM, Steis MR, Waller JL, Inouye SK. Delirium superimposed on dementia is associated with prolonged length of stay and poor outcomes in hospitalized older adults. J Hosp Med. 2013;8(9):500-505.

8. Lyketsos CG, Sheppard JM, Rabins PV. Dementia in elderly persons in a general hospital. Am J Psychiatry. 2000;157(5):704-707.

9. Yaffe K, Fox P, Newcomer R, et al. Patient and caregiver characteristics and nursing home placement in patients with dementia. JAMA. 2002;287(16):2090-2097.

10. Stern Y, Tang MX, Albert MS, et al. Predicting time to nursing home care and death in individuals with Alzheimer disease. JAMA. 1997;277(10):806-812.

11. Hebert LE, Weuve J, Scherr PA, Evans DA. Alzheimer disease in the United States (2010-2050) estimated using the 2010 census. Neurology. 2013;80(19):1778-1783.

12. Phelan EA, Debnam KJ, Anderson LA, Owens SB. A systematic review of intervention studies to prevent hospitalizations of community-dwelling older adults with dementia. Med Care. 2015;53(2):207-213.

13. Pimouguet C, Lavaud T, Dartigues JF, Helmer C. Dementia case management effectiveness on health care costs and resource utilization: a systematic review of randomized controlled trials. J Nutr Health Aging. 2010;14(8):669-676.

14. Caverly TJ, Hayward RA, Burke JF. Much to do with nothing: microsimulation study on time management in primary care. BMJ. 2018;363:k4983.

15. Bynum JP. The long reach of Alzheimer's disease: patients, practice, and policy. Health Aff (Millwood). 2014;33(4):534-540.

16. Kales HC, Kim HM, Zivin K, et al. Risk of mortality among individual antipsychotics in patients with dementia. Am J Psychiatry. 2012;169(1):71-79.

17. Kales HC, Valenstein M, Kim HM, et al. Mortality risk in patients with dementia treated with antipsychotics versus other psychiatric medications. Am J Psychiatry. 2007;164(10):1568-1576. quiz 1623.

18. Maust DT, Kim HM, Seyfried LS, et al. Antipsychotics, other psychotropics, and the risk of death in patients with dementia: number needed to harm. JAMA Psychiat. 2015;72(5):438-445.

19. Agency for Healthcare Research and Quality. Prevention quality indicators technical specifications, v.5.0, March 2015. http://www.qualityindicators. ahrq.gov/Modules/PQI_TechSpec.aspx. Accessed March 19, 2015.

20. Bynum JP, Rabins PV, Weller W, Niefeld M, Anderson GF, Wu AW. The relationship between a dementia diagnosis, chronic illness, Medicare expenditures, and hospital use. J Am Geriatr Soc. 2004;52(2):187-194.

21. Rudolph JL, Zanin NM, Jones RN, et al. Hospitalization in communitydwelling persons with Alzheimer's disease: frequency and causes. J Am Geriatr Soc. 2010;58(8):1542-1548.

22. Lemke KW, Weiner JP, Clark JM. Development and validation of a model for predicting inpatient hospitalization. Med Care. 2012;50(2):131-139.

23. Gao J, Moran E, Li Y-F, Almenoff PL. Predicting potentially avoidable hospitalizations. Med Care. 2014;52(2):164-171.

24. Russ TC, Parra MA, Lim AE, Law E, Connelly PJ, Starr JM. Prediction of general hospital admission in people with dementia: cohort study. Br J Psychiatry. 2015;206(2):153-159.

25. Maust DT, Kales HC, McCammon RJ, Blow FC, Leggett A, Langa KM. Distress associated with dementia-related psychosis and agitation in relation to healthcare utilization and costs. Am J Geriatr Psychiatry. 2017;25(10):1074-1082.

26. Maust DT, Langa KM, Blow FC, Kales HC. Psychotropic use and associated neuropsychiatric symptoms among patients with dementia in the USA. Int J Geriatr Psychiatry. 2016;24:S151-S153.

27. McCarthy JF, Bossarte RM, Katz IR, et al. Predictive modeling and concentration of the risk of suicide: implications for preventive interventions in the US Department of Veterans Affairs. Am J Public Health. 2015;105(9):1935-1942.

28. Meurer WJ, Tolles J. Logistic regression diagnostics: understanding how well a model predicts outcomes. JAMA. 2017;317(10):1068-1069.

29. Amjad H, Wong SK, Roth DL, et al. Health services utilization in older adults with dementia receiving care coordination: the MIND at home trial. Health Serv Res. 2017;23(3):271.

30. Callahan CM, Boustani MA, Unverzagt FW, et al. Effectiveness of collaborative care for older adults with Alzheimer disease in primary care: a randomized controlled trial. JAMA. 2006;295(18):2148-2157.
31. Mittelman MS, Ferris SH, Shulman E, Steinberg G, Levin B. A family intervention to delay nursing home placement of patients with Alzheimer disease. A randomized controlled trial. JAMA. 1996;276(21):1725-1731.

32. Mittelman MS, Haley WE, Clay OJ, Roth DL. Improving caregiver wellbeing delays nursing home placement of patients with Alzheimer disease. Neurology. 2006;67(9):1592-1599.

33. Klug MG, Halaas GW, Peterson ML. North Dakota assistance program for dementia caregivers lowered utilization, produced savings, and increased empowerment. Health Aff (Millwood). 2014;33(4):605-612.

34. Counsell SR, Callahan CM, Clark DO, et al. Geriatric care management for low-income seniors: a randomized controlled trial. JAMA. 2007;298(22): 2623-2633.

35. Kharrazi H, Anzaldi LJ, Hernandez L, et al. The value of unstructured electronic health record data in geriatric syndrome case identification. J Am Geriatr Soc. 2018;60:896-899.

36. Kim DH, Schneeweiss S. Measuring frailty using claims data for pharmacoepidemiologic studies of mortality in older adults: evidence and recommendations. Pharmacoepidemiol Drug Saf. 2014;23(9):891-901.

37. Boult C, Dowd B, McCaffrey D, Boult L, Hernandez R, Krulewitch H. Screening elders for risk of hospital admission. J Am Geriatr Soc. 1993;41(8): 811-817.

38. Coleman EA, Wagner EH, Grothaus LC, Hecht J, Savarino J, Buchner DM. Predicting hospitalization and functional decline in older health plan enrollees: are administrative data as accurate as self-report? J Am Geriatr Soc. 1998;46(4): 419-425.

39. Haas LR, Takahashi PY, Shah ND, et al. Risk-stratification methods for identifying patients for care coordination. Am J Manag Care. 2013;19(9): $725-732$.

40. Crane SJ, Tung EE, Hanson GJ, Cha S, Chaudhry R, Takahashi PY. Use of an electronic administrative database to identify older community dwelling adults at high-risk for hospitalization or emergency department visits: the elders risk assessment index. BMC Health Serv Res. 2010;10(1):338.

41. Inouye SK, Zhang Y, Jones RN, et al. Risk factors for hospitalization among community-dwelling primary care older patients: development and validation of a predictive model. Med Care. 2008;46(7):726-731.

42. Kales HC, Gitlin LN, Lyketsos CG. Assessment and management of behavioral and psychological symptoms of dementia. BMJ. 2015;350(mar02 7):h369.

43. Finegan MS, Gao J, Pasquale D, Campbell J. Trends and geographic variation of potentially avoidable hospitalizations in the veterans health-care system. Health Serv Manage Res. 2010;23(2):66-75.

44. McCall N, Harlow J, Dayhoff D. Rates of hospitalization for ambulatory care sensitive conditions in the Medicare+ choice population. Health Care Financ Rev. 2001;22(3):127-145.

45. Patel KK, Vakharia N, Pile J, Howell EH, Rothberg MB. Preventable admissions on a general medicine service: prevalence, causes and comparison with AHRQ prevention quality indicators-a cross-sectional analysis. J Gen Intern Med. 2016;31(6):597-601.

46. Hodgson K, Deeny SR, Steventon A. Ambulatory care-sensitive conditions: their potential uses and limitations. BMJ Qual Saf. 2019;28:429-433.

\section{SUPPORTING INFORMATION}

Additional Supporting Information may be found in the online version of this article.

Table S1: ICD-9-CM diagnoses used to define potentially preventable hospitalization (PPH) and non-Charlson medical and psychiatric conditions

Table S2: Complete demographic and clinical characteristics and 30-day and 1-year rates of PPH among the development cohort

Table S3: Coefficients of all variables in the final model in the development cohort

Table S4: Cumulative PPH rate and risk concentration from 1 month to 1 year by cut point of 30-day predicted $\mathrm{PPH}$ risk in the prediction cohort

Supplementary Material S1: Variable-defining ICD9-CM codes, characteristics, and model coefficients of the development cohort and PPH risk decay over 1 year. 